\title{
Direccionamiento estratégico de la nutrición clínica en un hospital de alta complejidad
}

\author{
Strategic address of clinical nutrition in a high-complex hospital \\ Direção estratégica da nutrição clínica em um hospital de alta complexidade
}

\author{
Esperanza Moncada Parada ${ }^{* *}$, Jhon Jairo Martínez Yacelga' \\ Recibido: 2 de junio de 2020. Aceptado para publicación: 15 de junio de 2020 \\ Primero en línea: 30 de junio de 2020 \\ https://doi.org/10.35454/rncm.v3n2.159
}

\section{Resumen}

El manejo exitoso de la nutrición hospitalaria requiere un abordaje interdisciplinario y un liderazgo que fomente la comunicación abierta entre las disciplinas. Para tener éxito, todos los miembros del equipo de salud tienen que entender la relevancia del cuidado nutricional para mejorar el pronóstico del paciente y el impacto financiero.

El equipo interdisciplinario debe estar articulado y capacitado, de manera que brinde al paciente las mejores opciones en cuanto a su tratamiento, pronóstico, monitoreo y evaluación de las situaciones particulares dadas por el cuadro clínico.

En esta interdisciplinaridad, los servicios de nutrición y dietética adquieren protagonismo considerando los múltiples beneficios evidenciados en los pacientes que reciben una aproximación nutricional desde las primeras horas de ingreso a las instituciones. Todos estos procedimientos para el tratamiento nutricional de los pacientes, deben estar soportados en políticas, programas y planes que sirven de sustento ideológico, teórico y preponderante para el correcto manejo nutricional en cada institución, considerando además la alimentación como un criterio transversal en todas las etapas de la vida y en el proceso de salud y enfermedad. El objetivo de este artículo es describir las acciones realizadas por la Empresa Social del Estado Hospital Universitario Erasmo Meoz, en el ámbito del direccionamiento estratégico para la prestación de servicios

\section{Summary}

Successful management of hospital nutrition requires an interdisciplinary team approach and a strong leadership that fosters open communication between disciplines. Moreover, all members of the health team must understand the relevance of nutritional care in improving patient prognosis and financial return. The interdisciplinary team must be articulated and trained in order to provide the best treatment, prognosis, monitoring and evaluation options according to each patient's clinical picture. Considering the multiple benefits evidenced in patients who receive nutritional support in the first hours after admission to the hospital, the nutrition and dietetic services acquire a central role in this interdisciplinarity.

Procedures for the nutritional treatment of patients must be supported by policies, programs and plans that serve as ideological, theoretical and fundamental support for the correct nutritional management in each health facility, and above all, nutrition must be considered a transverse principle at all stages of life, health and disease. The objective of this article is to describe the actions carried out by the Empresa Social del Estado Hospital Universitario Erasmo Meoz in the area of strategic direction for the provision of services related to nutrition and dietetics, in search for quality, continuous improvement, prevention of complications, reduction of treatment

\section{Resumo}

O êxito na gestão da utilização da nutrição hospitalar requer uma abordagem interdisciplinar e uma liderança que incentive a comunicação aberta entre as disciplinas. Para ter sucesso, todos os membros da equipe de saúde precisam entender a relevância do cuidado nutricional para melhorar o prognóstico do paciente e o impacto financeiro.

A equipe interdisciplinar deve ser articulada e treinada, de modo a oferecer ao paciente as melhores opções em termos de tratamento, prognóstico, monitorização e avaliação das situações particulares apresentadas pelo quadro clínico.

Nesta interdisciplinaridade, os serviços de nutrição e dietética adquirem protagonismo, considerando os múltiplos benefícios evidenciados nos pacientes que recebem uma abordagem nutricional desde as primeiras horas de internamento nas instituições. Todos esses procedimentos para o tratamento nutricional dos pacientes devem estar apoiados em políticas, programas e planos que sirvam de suporte ideológico, teórico e preponderante para o correto manejo nutricional em cada instituição, considerando além disso a alimentação como um critério transversal em todas as etapas da vida e no processo de saúde e de doença. O objetivo deste artigo é descrever as ações realizadas pela Empresa Social do Hospital Universitário Erasmo Meoz, na área de direcionamento estratégico para a prestação de serviços relacionados à nutrição e dietética em 
relacionados con la nutrición y dietética en búsqueda de la calidad, el mejoramiento continuo, la prevención de las complicaciones, disminución de los costos de tratamiento y estancia hospitalaria, el bienestar y seguridad del paciente.

Palabras clave: nutrición, nutrición clínica. costs and hospital stay, and patient wellbeing and safety.

Keywords: Nutrition; Clinical Nutrition. busca da qualidade, melhoria contínua, prevenção de complicações, redução de custos de tratamento e permanência hospitalar, o bem-estar e segurança do paciente.

Palavras-chave: nutrição, nutrição clínica.
Servicio de Nutrición y Dietética, E.S.E Hospital Universitario Erasmo Meoz, Cúcuta, Colombia.

\section{INTRODUCCIÓN}

El Hospital Universitario Erasmo Meoz, es producto de la Ley 39 de 1969, por medio de la cual se implementó el Plan Hospitalario Nacional en Colombia. Su creación como ente jurídico fue autorizada mediante Ordenanza 019 emanada de la Honorable Asamblea Departamental de Norte de Santander el día 2 de diciembre de 1986, con el nombre Erasmo Meoz. El 27 de noviembre de 2003, mediante Ordenanza 038, se modificó la denominación de la entidad quedando de la siguiente forma: E.S.E Hospital Universitario Erasmo Meoz, tal como se conoce en la actualidad ${ }^{(1)}$.

La Empresa Social del Estado (E.S.E.) Hospital Universitario Erasmo Meoz (HUEM), es una institución prestadora de servicios de salud (IPS) enfocada en el direccionamiento estratégico, con pilares fundamentales que constituyen las líneas de acción para la gestión y la prestación de los servicios de salud a la comunidad con influencia en el área departamental de Norte de Santander, el nororiente colombiano y el noroccidente venezolano y los departamentos de Arauca y Cesar. El direccionamiento estratégico está encauzado hacia la rentabilidad social y la sostenibilidad financiera, enmarcándose en la docencia e investigación, la atención humana y la seguridad del paciente, posicionándose como un referente en la región para la prestación de servicios de salud ambulatorios e intrahospitalarios de alta complejidad.

En la actualidad, la E.S.E. HUEM cuenta con áreas de urgencias, unidad de cuidados intensivos intermedios, cirugía, pediatría, neurocirugía, ortopedia, medicina interna, cirugía general y cuidados especiales. Con un total de 444 camas disponibles, esta capacidad se ve desbordaba en repetidas ocasiones debido a la gran afluencia de pacientes y usuarios que tiene la institución.
*Correspondencia: Esperanza Moncada Parada esperanzamoncadap@hotmail.com
El área de Nutrición y Dietética, parte del proceso misional y del subproceso "Apoyo Diagnóstico y Terapéutico", está regida por la Política de Atención Nutricional, la cual a su vez se encuentra articulada con el Programa de Atención Nutricional, adoptado por la institución mediante la Resolución No. 001519 del 15 de octubre de 2019, y que constituye un pilar fundamental en la orientación a la acción y el direccionamiento estratégico.

El objetivo de este artículo es, por un lado, brindar una perspectiva sobre el manejo y abordaje nutricional de los pacientes en un hospital público de alta complejidad en la capital de Norte de Santander, Colombia; y por otro lado, identificar el direccionamiento estratégico como un pilar fundamental para la prestación de servicios de salud relacionados con la Nutrición y la Dietética en una IPS de las características arriba descritas.

\section{EL ÁREA DE NUTRICIÓN Y DIETÉTICA Y SU COMPROMISO CON LA INSTITUCIÓN}

Desde el año 2012, la nutricionista Esperanza Moncada Parada lidera el área de Nutrición y Dietética la cual se encuentra en un proceso constante de renovación y actualización del conocimiento del equipo de Nutricionistas Dietistas. Esto se realiza en el marco de un adecuado abordaje nutricional, el mejoramiento continuo de todos los aspectos preponderantes en la atención nutricional, la búsqueda de la prestación de un mejor servicio mediante la fijación de metas en el plan de acción de la institucion como lo es la certificación de Buenas Prácticas de Manufactura (BPM), la adopción de lineamientos nacionales, y la formulación de políticas y programas institucionales. Estas acciones contribuyen al fortalecimiento de la gestión clínica, el 
mejoramiento de los pronósticos clínicos de los pacientes, la prevención de todo tipo de malnutrición y el empoderamiento de los profesionales con respecto al ejercicio de la nutrición clínica.

La gestión clínica evidencia que las diferentes disciplinas y servicios de un hospital deben dirigir sus procesos a ofrecer resultados costo-eficientes y a su vez vislumbrar la prestación de servicios con altos estándares de calidad en un entorno altamente competitivo. Las instituciones de salud requieren un programa de atención nutricional oportuna y eficiente que trabaje en el direccionamiento y abordaje nutricional, propendiendo por un manejo individualizado de los pacientes durante la estancia hospitalaria ${ }^{(2)}$.

La necesidad de modelos en atención nutricional radica en la magnitud del problema de malnutrición en los hospitales, el cual es de dimensión mundial. Como ejemplo tenemos que en Europa en el año 2003, el Comité de Ministros del Consejo Europeo, por consenso publicó la Resolución RESAP "Resolución Sobre Alimentación y Atención Nutricional en Hospitales" con el fin de elevar el nivel de atención en salud de los consumidores en los centros asistenciales, teniendo como referente que el acceso a una variedad segura y saludable de los alimentos es un derecho humano que se debe garantizar durante la atención ${ }^{(3)}$.

Desde el año 2012, el área de Nutrición y Dietética, jalona procesos de mejoramiento continuo que permiten brindar servicios de calidad a usuarios y pacientes de la E.S.E. HUEM; es por eso, que se adelantan acciones en el marco de los procesos misionales y de acreditación institucional a la que esta IPS se ha postulado.

En el ámbito de la Nutrición Clínica, se cuenta con una amplia gama de políticas, resoluciones, protocolos, guías, procedimientos, manuales, y otros documentos que sirven como sustento en el direccionamiento estratégico de nuestra área ${ }^{(4)}$. Lo anteriormente enunciado, está fundamentado en criterios de diferentes referentes de la Nutrición Clínica, basados en evidencia científica internacional y nacional como lo son la Federación Latinoamericana de Terapia Nutricional, Nutrición Clínica y Metabolismo (FELANPE), la Sociedad Europea de Nutrición Clínica y Metabolismo (ESPEN), Sociedad Americana de Nutrición Parenteral y Enteral (ASPEN), Asociación Colombiana de Nutrición Clínica (ACNC), Ministerio de Salud y Protección Social, y demás entes de consenso en el área, con el objetivo de articular y mantener un proceso constante de actualización.

\section{EL ABORDAJE NUTRICIONAL EN LA INSTITUCIÓN}

"El pasado 3 de mayo de 2019, en la ciudad de Cartagena, Colombia, 16 sociedades Iberoamericanas de la FELANPE, tras firmar la Declaración de Cartagena, se comprometieron a defender el derecho al cuidado nutricional y a luchar contra la malnutrición. Se trata de un instrumento no vinculante con una fuerza moral innegable, el cual compromete a las partes a aunar esfuerzos en esta lucha común."(3). "La Declaración de Cartagena, tiene como antecedente la Declaración de Cancún de 2008, que hace referencia por primera vez al derecho a la nutrición en el hospital en Latinoamérica. Además, se fundamenta en los instrumentos internacionales de derechos humanos y de bioética (la Declaración Universal de Derechos Humanos, el Pacto Internacional de Derechos Económicos, Sociales y Culturales, Declaración de Bioética y Derechos Humanos de la UNESCO), los cuales proporcionan una base sólida para sus objetivos." ${ }^{(5)}$.

La salud y la alimentación son preponderantes toda vez que tienen carácter de derecho fundamental; durante la dinámica del proceso salud - enfermedad y dentro de las instituciones prestadoras de servicios de salud, se deben garantizar dichos derechos que son inherentes al ser humano.

El proceso clínico se identifica por los procedimientos científicos y tecnológicos para establecer el diagnóstico y aplicar terapéuticas que requiere la condición patológica del paciente; en este punto la hotelería hospitalaria se convierte en un aspecto fundamental en la recuperación de la salud.

La E.S.E HUEM, consciente de la poca satisfacción de las expectativas del paciente respecto al servicio de alimentación, e identificada ésta como uno de los factores de desnutrición hospitalaria, el área de Nutrición y Dietética quiso intervenir de manera sistemática y meticulosa el servicio de alimentación. Para el logro de este propósito, se trazó como meta, no solo obtener la certificación en BPM, sino también alcanzar la satisfacción de las necesidades y expectativas de los pacientes, implementando el flexi menú, con la posibilidad de selección de dos opciones proteicas, durante la comida principal. Con esta estrategia, se han obtenido resultados favorables, los cuales son medidos a través de la aplicación de la encuesta de satisfacción. Este maniobra nos permitió ajustar nuestros objetivos a las necesidades del paciente en las diferentes etapas 
evolutivas de su enfermedad, máxime cuando reconocemos que ponen su vida en manos de personas que ellos confían en que les devolverán la salud y esta esperanza, de alguna manera, atenúa su ansiedad, convirtiendo así lo cualitativo tanto en valor como en el más diligente acto médico.

La operatividad del servicio de alimentación en nuestra institución, está soportada en un amplio conocimiento nutricional y clínico basado en la normatividad vigente y articulado directamente con la intervención médico nutricional, en donde el cuidado nutricional del paciente se convierte en parte fundamental del tratamiento de la enfermedad.

La difícil situación social por la que atraviesa nuestra región, en la actualidad agravada por un proceso de migración inesperado, nos convierte de manera contundente en una población más vulnerable nutricionalmente. Situación que se ve reflejada en altos índices de desnutrición, no solo en la población adulta sino, desafortunadamente, en la población pediátrica. Recordemos que en nuestra institución convergen la mayoría de la población del departamento de Norte de Santander, del nororiente colombiano, del noroccidente venezolano y de los departamentos de Arauca y Cesar.

Frente a la responsabilidad social que nos concierne, la recuperación de la salud, en nuestro accionar dentro de la E.S.E HUEM, se constituye en una columna fundamental, y es el motor que nos mueve cada día a buscar herramientas para el mejoramiento continuo en la calidad de la atención nutricional centrada en el paciente. Este accionar constante nos ha motivado a participar en actividades nacionales e internacionales como la Organización para la Excelencia en Nutrición (OES), el estudio multicéntrico internacional nutritionDay (Día de la Nutrición). También hemos buscado apoyo en la Asociación Colombiana de Nutrición Clínica y la Fundación Conocimiento; para implementar el protocolo de recuperación rápida en cirugía mayor (ERAS), liderado por la doctora Ángela María Navas Camacho, en la Clínica Reina Sofía.

Estas experiencias interinstitucionales por demás enriquecedoras, permiten ubicar en nuestros primeros objetivos los resultados de desempeño clínico, medirlos, analizarlos y compararnos con los mejores referentes, optimizando los procesos de atención y proporcionando instrumentos suficientes que nos han facultado la identificación y cierre de brechas con el fin de obtener mejores resultados, mejor atención y menores costos.

Recientemente nuestra Institución mediante la Resolución No. 01519 del 15 de octubre del año 2019, como sello que le da validez para la implementación y publicación de la misma, se define y se adopta la Política de Atención Nutricional, articulada con el modelo de cuidado nutricional, en el cual se enmarcan las acciones descritas en el apartado de este documento denominado "El abordaje nutricional en la institución" y constituye el fundamento estratégico e ideológico para la prestación del servicio en la institución.

La primera parte de esta política la soporta un amplio referente bibliográfico: leyes, resoluciones, decretos, actos administrativos, declaraciones, que son el sustento de temas relacionados con alimentación y nutrición en el plano internacional, nacional e institucional. Posterior a esto, cuenta con un apartado que no solo describe los seis pasos de nuestro modelo de cuidado de atención nutricional, sino que también establece responsabilidades y menciona a sus autores.

\section{EL MODELO DE CUIDADO NUTRICIONAL DE LA E.S.E. HUEM}

El proceso de cuidado nutricional tiene como objetivo gestionar las actividades técnicas, administrativas y científicas, relacionadas con la atención nutricional integral de forma segura, oportuna y pertinente a los pacientes. De esta manera se contribuye a la recuperación del estado de salud de los pacientes durante el proceso de atención médica, basados en la normatividad vigente con el fin de evitar y mitigar el riesgo en la prestación del servicio y satisfacer las necesidades de nuestros usuarios ${ }^{(2)}$.

Es de vital importancia para la garantía de la calidad de la prestación de servicios, un abordaje nutricional integral para cada uno de los pacientes desde el mismo momento en que ingresan a nuestra institución hospitalaria. Este modelo de cuidado nutricional, inicia durante las primeras 48 horas de estancia hospitalaria con la aplicación de la herramienta para la detección precoz del riesgo nutricional a todos los pacientes, niños y adultos, que ingresan por los servicios de urgencias, consulta externa y sala de partos (Figura 1).

Conocedores de la existencia de varias herramientas para el cribado nutricional, y que la ausencia de un patrón de oro aceptado es un punto de discusión, se realizó una búsqueda bibliográfica de publicaciones sobre comparación de herramientas de Tamizaje nutricional en las bases de datos de Pubmed, Scielo y Lilacs, desde 2007 a 2017. También se revisaron los artículos originales de los métodos con el fin de elegir una herramienta sencilla, rápida y de bajo costo, confiable y válida, sensi- 
ble y específica, fácil de diligenciar, aplicable a la mayoría de los pacientes y diseñada para incorporar datos asociados a la enfermedad, y disponibles al momento de la admisión. La Asociación Colombiana de Nutrición Clínica recomienda desde el año 2012 la utilización de la herramienta NRS 2002 (Nutritional Risk Score 2002) herrmienta que escogimos para adultos ${ }^{(6)}$. Para pediatría, se escogió la STAMP (Screening Tool for the Assessment of Malnutrition in Paediatrics); tanto la NRS 2002 como la STAMP son aplicadas por el personal médico en el momento del ingreso, y articuladas como paso obligatorio dentro de la historia clínica.

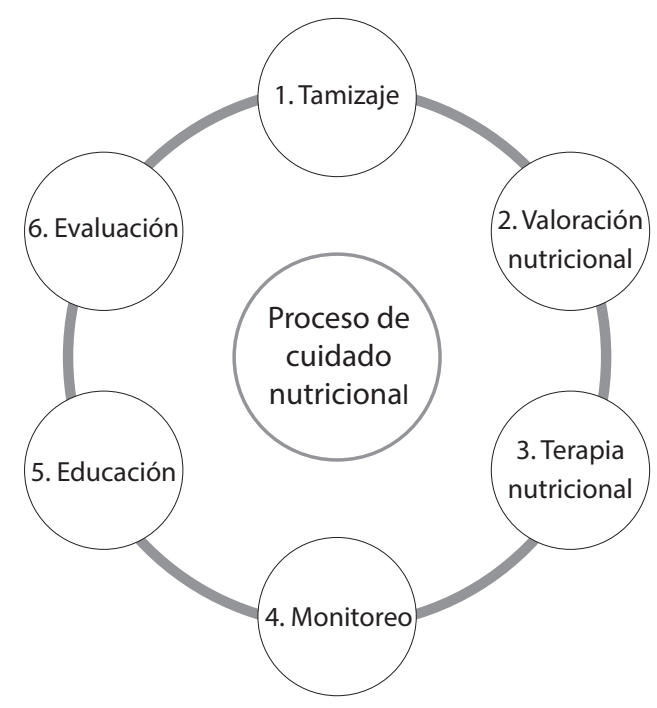

Figura 1. Proceso de cuidado nutricional de la E.S.E Hospital Universitario Erasmo Meoz, Cúcuta, Colombia. El proceso inicia con el tamizaje nutricional al ingreso hospitalario. Si el paciente está en riesgo se genera una interconsulta al servicio de nutrición y dietética la cual se realiza en las 24 horas siguientes. La valoración permite hacer un diagnóstico de la desnutrición y un plan nutricional. Esto conlleva a la terapia nutricional la cual puede ser a través de suplementos orales, nutrición enteral, nutrición parenteral o mixta. Los pacientes reciben educación sobre el proceso y al final se realiza una evaluación del proceso.

Una vez identificado el riesgo y considerando la secuencialidad del modelo de Cuidado Nutricional, el médico encargado de realizar el Tamizaje genera la solicitud de interconsulta por Nutrición y Dietética, la cual debe ser resuelta en un lapso de tiempo inferior a las 24 horas, y medida con el indicador denominado "oportunidad en la respuesta a interconsulta por la especialidad de nutrición y dietética", empleando los modelos de historia nutricional, diseñadas por el equipo de nutricionistas dietistas, y apoyados por la oficina de tecnología de la información y comunicación las cuales se encuentran disponibles en el aplicativo de registro de historia clínica institucional.

Como parte de la valoración nutricional, y para diagnosticar la desnutrición, se tienen en cuenta por lo menos dos o más criterios diagnósticos propuestos por $\operatorname{ASPEN}^{(7)}$.

- Ingesta energética insuficiente

- Pérdida de peso

- Pérdida de masa muscular

- Pérdida de grasa subcutánea

- Edemas localizados o generalizados que pueden enmascarar la pérdida de peso

- Disminución del estado funcional medido por la fuerza de prensión (uso del dinamómetro).

Complementado con parámetros alimentarios, bioquímicos, metabólicos, psicosociales, y estimación de los requerimientos energéticos individualizados según las comorbilidades del paciente y los objetivos de la implementación de una terapia nutricional especializada.

Así mismo, es importante mencionar que una terapia nutricional (modificación del plan nutricional) y un soporte nutricional (enteral o parenteral) debe tener en cuenta la condición clínica y el pronóstico de salud del paciente, con el objetivo de mantener las funciones inmunes siempre y cuando sea posible, previniendo las complicaciones metabólicas contribuyendo así a la disminución de la morbimortalidad, estancia hospitalaria y optimizando los costos para el sistema de salud.

En efecto, el monitoreo nutricional definido como el registro y evolución de la situación clínica del paciente, la adherencia al tratamiento nutricional, la identificación de los aspectos a mejorar y la corrección de las posibles falencias forman parte fundamental del seguimiento a la terapia implementada y constituye el paso cuarto del modelo adoptado por la institución, garantizando la continuidad del tratamiento y la satisfacción del paciente.

El paso cinco es el plan de educación sobre el cuidado nutricional para el momento de alta, con el objetivo de dar continuidad al tratamiento nutricional en casa y así mejorar de manera consecuente, la calidad del cuidado. La educación se hace extensiva a los familiares y cuidadores, contiene un objetivo claro, lenguaje sencillo, se realiza de manera escrita, y se tiene en cuenta la expectativa de vida. Se brinda al paciente y cuidador información específica en una consulta o cita de seguimiento, en caso de que la requiera. Finalmente, se realiza una sencilla evaluación para asegurar que la información fue entendida, de lo contario, se vuelve a educar. 
Para cerrar el ciclo del modelo de cuidado nutricional, correspondiente al paso sexto, seguimiento postegreso según el protocolo institucional, por medio del cual se establece comunicación con los pacientes, validando la información suministrada por el equipo de salud y verificando la adherencia del paciente a su tratamiento. El apoyo de los Nutricionistas Dietistas en formación de la Universidad de Pamplona, quienes realizan sus prácticas profesionales enmarcadas en el Convenio Docencia - Servicio, ha sido fundamental para el desarrollo del modelo.

Para dar cumplimiento al artículo 4 de la Resolución No 01519 del 15 de octubre de 2019, estamos en la etapa de la socialización y divulgación de la política, para que sea conocida por todo el personal que labora en la Institución.

\section{GRUPO DE SOPORTE METABÓLICO Y NUTRICIONAL (GSMN)}

El concepto de los grupos de Soporte Metabólico y Nutricional para la administración de nutrición enteral y parenteral se desarrolló durante la década del 80. Por esa época se produjeron varios trabajos que demostraban los beneficios de conformar equipos de soporte a nivel hospitalario. La Asociación Colombiana de Nutrición Clínica, desde su creación impulsó la creación y el mantenimiento de los mismos, siendo una iniciativa que se ha mantenido hasta el momento ${ }^{(8)}$.

Los GSMN contribuyen en gran medida a brindar una atención nutricional adecuada y oportuna en casos especiales, teniendo en cuenta un criterio interdisciplinar en el momento del abordaje nutricional y la toma de decisiones. Como institución prestadora de servicios de salud de alta complejidad es necesario contar con dicho grupo. Por lo tanto, se adelantan acciones tendientes a la conformación del mismo.

Mediante la Resolución N 01624 del 01 de noviembre de 2019, se define y se conforma el GSMN del Hospital Universitario Erasmo Meoz. Este acto administrativo fue desarrollado mediante un consenso entre el equipo de Nutrición y Dietética, el Líder Apoyo Dignóstico y Terapéutico, Planeación, Subgerencia de Servicios de Salud y el Asesor Jurídico de la Subgerencia, como una herramienta idónea para seleccionar a los pacientes en riesgo nutricional. Esto es considerado un factor clave que permite el uso apropiado de los recursos y el establecimiento de límites, la asignación de funciones a cada profesional, la prevención de las complicaciones, y la decisión con mejor relación costo-efectividad en el tratamiento en búsqueda del beneficio del paciente.

\section{LA INSTITUCIÓN PÚBLICA Y LA IMPLEMENTACIÓN DE POLÍTICAS Y PROGRAMAS RELACIONADOS CON NUTRICIÓN Y DIETÉTICA}

Teniendo en cuenta la normativa establecida en Colombia, dentro del ámbito nutricional se destaca el lineamiento para el tratamiento de la desnutrición aguda moderada y severa en población infantil de 0 a 59 meses de edad, el cual es de obligatorio cumplimiento y constituye la hoja de ruta para el tratamiento de esta patología ${ }^{(9)}$. Brinda herramientas e información importantes para el abordaje intrahospitalario y ambulatorio de la desnutrición como también establece otras consideraciones para el diagnóstico y la valoración nutricional. Es importante resaltar que al momento no existen políticas públicas ni lineamientos que aborden la desnutrición en el paciente adulto.

Considerando la importancia que reviste el lineamiento antes mencionado, la E.S.E. HUEM lo adoptó mediante la Resolución No. 01587 de 28 de octubre de 2019. Estos lineamientos se dieron a conocer a todo el personal involucrado en la atención de pacientes pediátricos y se implementaron todas las consideraciones allí establecidas.

La experiencia de formulación de este tipo de documentos es importante tanto para los profesionales de nutrición y dietética, como para el equipo interdisciplinar que participa activamente en estos procesos; cabe resaltar la colaboración y apoyo de los profesionales pertenecientes a las dependencias de Subgerencia en Salud, Apoyo Diagnóstico y Terapéutico y Planeación por su compromiso con la Institución, con los procesos de acreditación y la formulación de directrices para el direccionamiento estratégico de la institución hospitalaria.

\section{CONCLUSIONES}

El bienestar y salud de los pacientes, y la búsqueda de la prestación de un servicio de calidad, son las motivaciones principales para establecer un direccionamiento estratégico articulado con las demás dependencias de la institución. El direccionamiento estratégico mediante la formulación de actos administrativos, resoluciones, políticas, decretos y otros documentos es de vital importancia en una organización para el planteamiento de un horizonte claro y definido. La nutrición clínica necesita 
el direccionamiento estratégico para fomentar en la institución la adopción de modelos de cuidado nutricional en el marco de la garantía para la prestación de servicios. La formulación de políticas y programas en el sector salud requiere la participación de una amplia gama de profesionales de la salud, además de una recopilación de conocimientos y la voluntad de los dirigentes de la institución prestadora de servicios de salud.

\section{Financiación}

El presente trabajo no tuvo financiación.

\section{Declaración de conflicto de intereses}

Los autores declaran la inexistencia de conflictos de interés en relación con este artículo.

\section{Declaración de autoría}

Los autores contribuyeron en partes iguales a la escritura, revisión y documentación de la presente revisión. Los autores validaron la versión final del documento.

\section{Referencias bibligráficas}

1. Reseña Histórica. Marco legal y actos administrativos de creación. (Cosultado el 30 de abril 2020) Disponbile en: http:// www.herasmomeoz.gov.co/index.php/nuestra-empresa/ resena-historica.

2. Pinzón-Espitia OL, Pardo OviedoJM, González RodríguezJL. Modelo Méderi de gestión nutricional hospitalaria. Nutr Hosp. 2015;32(4):1802-1807. doi:10.3305/nh.2015.32.4.9196.
3. Council of Europe Committee of Ministers. Resolution $\operatorname{RESAP}(2003) 3$ on food and nutritional care in hospitals. Strasbourg (France); 2003 (Consultado el 8 de febrero de 2020]) Disponible en: http://www.Ake-Nutrition. At/Uploads/Media/Resolution_Of_The_Council_Of_ Europe_English.pdf.

4. Ministerio de Salud de Colombia. Prevención de la malnutrición o desnutrición paquetes instruccionales guía técnica "buenas prácticas para la seguridad del paciente en la atención en salud”. (Consultado el 30 de abri). Disponible en : https:// www.minsalud.gov.co/sites/rid/Lists/BibliotecaDigital/ RIDE/DE/CA/prevenir-la-malnutricion-o-desnutricion.pdf.

5. Cardenas D, Bermúdez CE, Echeverri S, et al. Declaración de Cartagena. Declaración Internacional sobre el Derecho al Cuidado Nutricional y la Lucha contra la Malnutrición. Nutr Hosp. 2019;36(4):974-80. doi:10.20960/nh.02701.

6. Cardenas D, Angarita C, Posada C, Osorio L, Rodríguez M, Echeverri S, et al. Recomendaciones de la Asociación Colombiana de Nutrición Clínica para la tamización nutricional en Colombia. Rev Col Metabol Nutr Clín. 2012;3(1):23-31.

7. White JV, Guenter P, Jensen G, Malone A, Schofield M; Academy Malnutrition Work Group, A.S.P.E.N. Malnutrition Task Force, A.S.P.E.N. Board of Directors. J Acad Nutr Diet. 2012;112(5):730-8.

8. Savino P. Desnutrición hospitalaria: grupos de soporte metabólico y nutricional. Rev. Colomb. Cir. 2012;27:46-54.

9. Ministerio de Salud de Colombia, UNICEF. Lineamiento para el manejo integrado de la desnutrición aguda moderada y severa en niños y niñas de 0 a 59 meses de edad. Colombia, 2020. (Consultado el 2 de junio 2020) Disponible en : https:// www.minsalud.gov.co/sites/rid/Lists/BibliotecaDigital/ RIDE/VS/PP/lineamiento-desnutricion-aguda-minsaludunicef-final.pdf. 